\title{
Virtual Constraints Based Control Design of an Inclined Translational Oscillator with Rotational Actuator System
}

\author{
Bingtuan Gao, ${ }^{1,2}$ Chuande Liu, ${ }^{1}$ and Hongtai Cheng ${ }^{3}$ \\ ${ }^{1}$ School of Electrical Engineering, Southeast University, Nanjing 210096, China \\ ${ }^{2}$ Jiangsu Key Laboratory of Smart Grid Technology and Equipment, Nanjing 210096, China \\ ${ }^{3}$ School of Mechanical Engineering and Automation, Northeastern University, Shenyang 110819, China \\ Correspondence should be addressed to Bingtuan Gao; gaobingtuan@seu.edu.cn \\ Received 16 April 2015; Revised 12 July 2015; Accepted 21 July 2015 \\ Academic Editor: Georges Kouroussis
}

Copyright (C) 2015 Bingtuan Gao et al. This is an open access article distributed under the Creative Commons Attribution License, which permits unrestricted use, distribution, and reproduction in any medium, provided the original work is properly cited.

\begin{abstract}
Translational oscillator with rotational actuator (TORA) system, whose translational and rotational movements occur in horizontal planes, is a benchmark of underactuated mechanical systems for studying of control techniques. Currently, the research work of the benchmark mainly focuses on stabilizing control of equilibrium points of the dynamical system. The problem of steering TORA to arbitrary points in its state space is rarely studied. In this paper, the movements of the TORA system are extended to inclined planes and dynamics of the inclined TORA system is presented firstly. Following that, a trajectory tracking control method based on virtual constraints is proposed to steer the oscillations of the inclined TORA system. A virtual constraints based method is presented to generate periodic trajectories which pass through desired point; and a Lyapunov based control design is proposed to track the generated trajectories. Finally, the performance and feasibility of the proposed control design methodology are illustrated and analyzed according to numerical simulations.
\end{abstract}

\section{Introduction}

The translational oscillator with rotational actuator (TORA) system consists of an unactuated translational oscillation cart and an actuated eccentric rotor attached to the cart, which is a benchmark of underactuated mechanical systems [1]. Underactuated mechanical systems were defined as a class of systems having fewer control inputs than the number of configuration variables [2-4]. It is interesting and challenging to analyze and control design underactuated mechanical systems. Currently, the control objectives of TORA system are to employ the control input torque acting or the rotor to stabilize both the translational position of the unactuated oscillating cart and the rotational position of the actuated eccentric rotor.

Before several interesting and effective control design methods [5, 6] presented in the special issue [1] entitled "International Journal of Robust and Nonlinear Control" in 1998, Jankovic et al. [7] have already presented several cascade-based effective controllers for the TORA system in 1996. Lee and Chang [8] combined an adaptive backstepping control scheme based on a wavelet-based neural network and a compensated controller to improve the closed-loop control performance of TORA system. Petres et al. [9] studied approximation and complexity trade-off by tensor production model transformation in control design with TORA system. In [10], an equivalent-input-disturbance method was proposed for TORA system with two steps. Moreover, several control design techniques resulting in controllers with only rotor angle feedback were presented in [11-13], which could be easier to be implemented in practice.

In the literature, the studied TORA system lies on horizontal planes, and there are infinite equilibriums of the rotor. In [14], dynamics and control design of a TORA system with rotating motion in a vertical plane were studied. Due to the gravity effect in the dynamics, there are only two equilibriums of the rotor, and a simple PD (proportional derivative) controller was designed to stabilize the cart oscillation while bringing the rotor angle to its downward equilibrium. Avis et al. [15] compared energy-based controller and sliding mode based controller designed for TORA system with rotating motion in a vertical plane. Since there does not exist absolute horizontal or vertical plane in practical 
implementations, the movements of TORA system will be in inclined planes. Generalized dynamics and controllability analysis of inclined TORA systems have been presented in detail in [16]. The same group has also extended translational oscillation of the TORA system to two-dimensional perpendicular translational movements, and passivity-based control design [17] was employed to stabilize the 2-dimensional TORA system to its controllable equilibriums.

In all the above-mentioned papers, the research work of underactuated TORA has been focused on stabilizing control of equilibrium points of the dynamical system. How about, inversely, forcing the oscillation to some points or tracking a trajectory in state space of the underactuated TORA system? Although forcing stable oscillations via feedback and analysis of such oscillations in fully actuated mechanical systems is an old area of research, the problem of forcing oscillations via feedback in underactuated mechanical systems has just attracted some attention [18]. Tracking control of periodic trajectory has been studied for underactuated pendulum-like systems due to its internal simplicity as early as in 1995 [19]. In the paper, a group of periodic trajectories for pendulumlike systems were generated by regulating the energy after studying the relationship between the state variables and the system energy. Aiming at mechanical systems with the number of independent actuators smaller than the number of degrees of freedom by one, Shiriaev et al. [18] proposed a systematic constructive tool for generation and orbital stabilization of periodic trajectories. The main idea is to impose virtual holonomic constraints [20] to the original system by using control inputs so that the original complicated dynamics can be reduced to the so-called virtual limit system, which is easier to analyze and control. This technique was applied into several underactuated systems, such as cart-pole system $[18,20]$ and Furuta pendulum $[21,22]$. In [23], the virtual constraints based algorithm was improved to generate periodic trajectories passing through arbitrary points in its state space. The method was then implemented successfully to the underactuated Acrobot.

In this paper, following our previous work [23], virtual constraints based method will be employed to generate periodic trajectories of the inclined TORA system, and a Lyapunov based control scheme is proposed to track the generated trajectories. As a result, the closed-loop underactuated TORA system will be able to oscillate through the starting points and the desired points in state space. Comparing with our previous work $[16,17]$ on TORA system which is on stabilizing control of equilibrium points of the nonlinear dynamical system, this paper presents a virtual constraint based control scheme for the inclined TORA system to steer the system oscillating according to desired trajectories passing through desired points. To the best of our knowledge, it is the first study on oscillating trajectory tracking control for TORA system and especially for the general inclined case.

The rest of the paper is organized as follows. Dynamics of the inclined TORA is presented in Section 2. Based on the dynamics, virtual constraints based method is employed to generate periodic trajectories for inclined TORA system in Section 3. After that, a Lyapunov based control scheme is adopted to design the trajectory tracking controller for the system in Section 4. Simulation results and discussions will be elaborated in Section 5. And conclusions are presented in the last section.

\section{Dynamics of Inclined TORA System}

The system shown in Figure 1 represents a comparison of the benchmark system TORA on the horizontal plane [1] and inclined TORA system [16]. For the TORA system on the horizontal plane, a coordinate frame oxyz is attached to the TORA system, with the origin at the center of the cart when the spring is with free status. The $x$-axis is along the translational motion of the cart, and the $z$-axis is perpendicular to the plane of the TORA system. Let $g$ be the gravity constant, and its direction is the negative $z$. For the inclined TORA system, another world frame OXYZ is defined for the slope according to the definition of oxyz. The movements of TORA system occur in the inclined plane oxy having an angle $\beta$ with respect to the horizontal plane $O X Y$; and inclining direction of the TORA system is denoted with $\gamma$. The inclined angle $\beta$ of the slope could be caused by tilted installation plane or assembly process, which can be defined as $\beta \in\left[0^{\circ}, 90^{\circ}\right]$. The inclining direction angle $\gamma$ of the inclined TORA system could be selected arbitrarily in $\gamma \in\left[0^{\circ}, 360^{\circ}\right)$.

Parameters of the TORA system are defined according to the coordinate frame oxyz similarly as in previous literatures $[1,16]$. The cart of mass $M$ is connected to a fixed base by a linear spring with stiffness $k$. The cart is constrained to have one-dimensional translational movement with $x$ denoting the travel distance. The actuated rotor attached to the cart has mass $m$ and moment of inertia $I$ about its center of mass; and the eccentric distance of the rotor is $r$. Control input torque applied to the rotor is denoted as $\tau$. Let $x$ and $\dot{x}$ denote the translational position and velocity of the cart, respectively; and let $\theta$ and $\dot{\theta}$ denote angular position and the angular velocity of the rotor, respectively.

The total kinetic energy $T$ and potential energy $P$ of the inclined TORA system can be calculated as

$$
\begin{aligned}
T= & \frac{1}{2}(M+m) \dot{x}^{2}+m r \cos \theta \dot{x} \dot{\theta}+\frac{1}{2}\left(m r^{2}+I\right) \dot{\theta}^{2}, \\
P= & \frac{1}{2} k x^{2}+(M+m) g x \cos \gamma \sin \beta \\
& +m g r \sin (\gamma+\theta) \sin \beta .
\end{aligned}
$$

Therefore, the Lagrangian $L$ can be calculated as

$$
L=T-P .
$$

By choosing $x$ and $\theta$ as the generalized coordinates and $\tau$ as the generalized force, based on Lagrange equations, dynamics of motion for the inclined TORA system can be derived as

$$
\begin{aligned}
& (M+m) \ddot{x}+m r \cos \theta \ddot{\theta}-m r \sin \theta \dot{\theta}^{2}+k x \\
& \quad+(M+m) g \cos \gamma \sin \beta+N_{x}=0, \\
& m r \cos \theta \ddot{x}+\left(m r^{2}+I\right) \ddot{\theta}+m g r \cos (\gamma+\theta) \sin \beta \\
& \quad+N_{r}=\tau,
\end{aligned}
$$




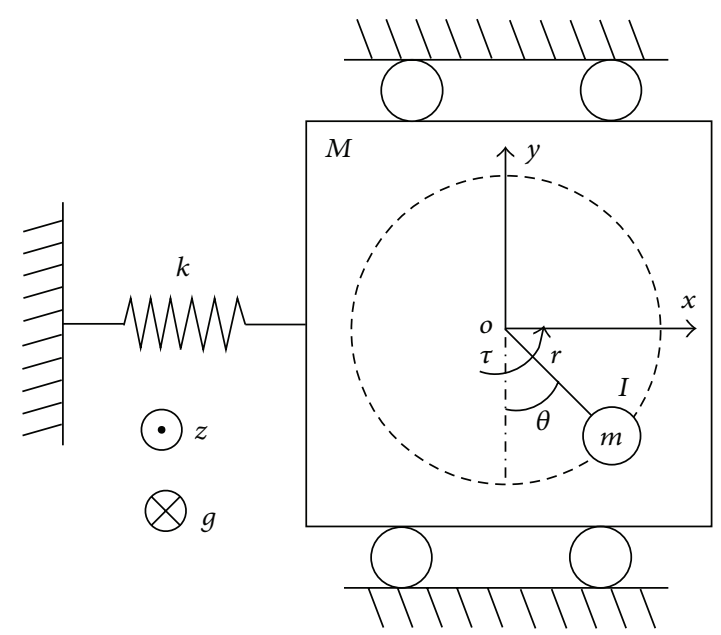

(a)

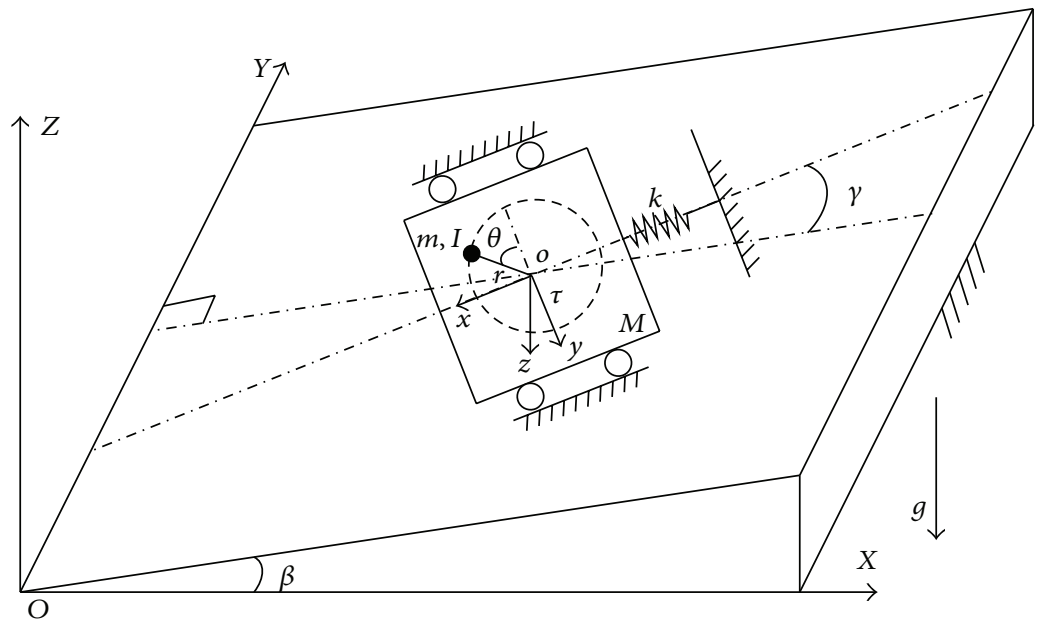

(b)

FIgURE 1: The configuration of TORA system: (a) TORA system in a horizontal plane and (b) TORA system on a slope.

where $N_{x}$ and $N_{r}$ denote the disturbance inputs acting on the translational moving cart and rotating rotor, respectively.

In compact matrix form, the above two equations can be rewritten as

$$
\mathbf{M}(\mathbf{q}) \ddot{\mathbf{q}}+\mathbf{C}(\mathbf{q}, \dot{\mathbf{q}}) \dot{\mathbf{q}}+\mathbf{G}(\mathbf{q})+\mathbf{N}=\mathbf{U},
$$

where $\mathbf{q}=\left(q_{1}, q_{2}\right)^{\mathrm{T}}=(x, \theta)^{\mathrm{T}}$ is the variable vector and $\mathbf{M}(\mathbf{q})$, $\mathbf{C}(\mathbf{q}, \dot{\mathbf{q}}), \mathbf{G}(\mathbf{q}), \mathbf{N}$, and $\mathbf{U}$ represent inertia matrix, Coriolis and centrifugal force matrix, potential matrix, disturbance force vector, and control input vector of the inclined TORA system, respectively:

$$
\begin{aligned}
\mathbf{M}(\mathbf{q}) & =\left[\begin{array}{ll}
m_{11} & m_{12} \\
m_{21} & m_{22}
\end{array}\right]=\left[\begin{array}{cc}
M+m & m r \cos \theta \\
m r \cos \theta & m r^{2}+I
\end{array}\right], \\
\mathbf{C}(\mathbf{q}, \dot{\mathbf{q}}) & =\left[\begin{array}{cc}
0 & -m r \dot{\theta} \sin \theta \\
0 & 0
\end{array}\right], \\
\mathbf{G}(\mathbf{q}) & =\left[\begin{array}{c}
k x+(M+m) g \cos \gamma \sin \beta \\
m g r \cos (\gamma+\theta) \sin \beta
\end{array}\right],
\end{aligned}
$$

$$
\begin{aligned}
& \mathbf{N}=\left[\begin{array}{l}
N_{x} \\
N_{r}
\end{array}\right], \\
& \mathbf{U}=\left[\begin{array}{l}
0 \\
\tau
\end{array}\right] .
\end{aligned}
$$

From dynamics (4), one can see straightforwardly that $\theta$ is the actuated variable and $x$ is the unactuated variable. The configuration variables $x$ and $\theta$ of the underactuated inclined TORA system have to be controlled by its only control input torque $\tau$. Another observation is that when $\beta=0$, that is, the TORA system on a horizontal plane, the inclined TORA system dynamics (4) will be deduced as

$$
\begin{array}{r}
(M+m) \ddot{x}+m r \cos \theta \ddot{\theta}-m r \sin \theta \dot{\theta}^{2}+k x+N_{x}=0, \\
m r \cos \theta \ddot{x}+\left(m r^{2}+I\right) \ddot{\theta}+N_{r}=\tau
\end{array}
$$

which is the dynamics of the benchmark TORA system. 
By neglecting the disturbance force Matrix $\mathbf{N}$ and letting $\tau=0$ in the inclined TORA system dynamics (4), the equilibriums of the system can be derived as

$$
\begin{aligned}
& x_{e}=\frac{(M+m) g \cos \gamma \sin \beta}{k} \\
& \theta_{e}=\left(\frac{2 n+1}{2}\right) \pi-\gamma, \quad n=0, \pm 1, \pm 2, \ldots,
\end{aligned}
$$

where we can see that the inclined TORA system has two types of equilibriums, that is, up equilibriums with high potential energy of gravity and down equilibriums with low potential energy of gravity.

\section{Virtual Constraints Based Trajectory Generation}

According to equilibrium (8) of the inclined TORA system, the up equilibriums are self-unstable which are not interesting. In this paper, we will extend the down equilibriums to arbitrary configuration space points. To do that, we identify the form of desired point $\left[q_{1}, q_{2}, \dot{q}_{1}, \dot{q}_{2}\right]=\left[q_{1 d}, q_{2 d}, 0,0\right]$. And because of the simplicity and stability of the periodic orbits, periodic trajectories will be focused on. The objective of virtual constraints based method for the inclined TORA system is to generate periodic trajectories which pass through desired point $\left[q_{1 d}, q_{2 d}, 0,0\right]$ firstly. The meaning of virtual constraints is defined contrary to physical constraints which exist practically, while the function of virtual constraints for TORA system is to realize that trajectory of the dynamical system begins from the starting point and stabilizes to the designed periodic trajectory passing through the desired point.

3.1. Virtual Constraints Design. The trajectories of the cart and the rotor of the inclined TORA system can be written as

$$
\begin{aligned}
& q_{1}=\varphi_{1}(t), \\
& q_{2}=\varphi_{2}(t) .
\end{aligned}
$$

For the fully actuated mechanical system, the above trajectories can be tracked by decoupling the coordinates and the control inputs. However, for the underacutated TORA system, the method fails because the control inputs are fewer than the configuration variables. By eliminating time $t$ and choosing a coordinate $q_{2}$, the two trajectory functions (9) can be transformed into the following single function:

$$
q_{1}=\varphi\left(q_{2}\right)
$$

Single equation (10) cannot fully define a trajectory. The missing equation could be an explicit/implicit function with respect to time, such as

$$
\varphi_{2}\left(q_{2}, \dot{q}_{2}\right)=0
$$

Now, together with (11), function (10) is equivalent to the two time functions (9). It is easy to verify that function (10) can be seen as holonomic constraint. If it is imposed on the original system using the control inputs, the original system becomes a one-dimensional inclined dynamic system. Additionally, if time function (11) is compatible with the dynamic system, the corresponding trajectory is able to be tracked. Since the holonomic constraint does not physically exist, it is called virtual constraint.

To find out the simplest virtual constraint of the two points, that is, starting point and desired point of the period orbit, it comes to an imaginary line constrained by the two points straightforwardly, which can be expressed as

$$
a_{1} q_{1}+a_{2} q_{2}+a_{3}=0,
$$

where $a_{1} \neq 0$ or $a_{2} \neq 0$. For given starting and desired points $\left[x_{e}, \theta_{e}\right]$ and $\left[x_{d}, \theta_{d}\right]$, without loss of generality, line constraint (12) can be rewritten as

$$
q_{1}=a q_{2}+b
$$

where

$$
\begin{aligned}
& a=\frac{x_{d}-x_{e}}{\theta_{d}-\theta_{e}}, \\
& b=\frac{x_{e} \theta_{d}-x_{d} \theta_{e}}{\theta_{d}-\theta_{e}} .
\end{aligned}
$$

3.2. Trajectory Calculation. By combining inclined TORA dynamics (4) and virtual constraint (13), the system zero dynamics can be calculated as [18]

$$
\eta\left(q_{2}\right) \ddot{q}_{2}+\sigma\left(q_{2}\right) \dot{q}_{2}^{2}+\omega\left(q_{2}\right)=0,
$$

where

$$
\begin{aligned}
& \eta\left(q_{2}\right)=a(M+m)+m r \cos q_{2}, \\
& \sigma\left(q_{2}\right)=-m r \sin q_{2}, \\
& \omega\left(q_{2}\right)=k\left(a q_{2}+b\right)+(M+m) g \cos \gamma \sin \beta .
\end{aligned}
$$

One can see straightforwardly that, in the role of virtual constraints, the inclined TORA system will become a singledimensional dynamic system, which is described by a second order differential equation. Therefore, corresponding to every initial state $\left(q_{0}, \dot{q}_{0}\right)$, there always exist determined integral curves, which can be written as $\psi\left(q_{2}, \dot{q}_{2}, q_{0}, \dot{q}_{0}\right)$. If the initial state $\left(q_{0}, \dot{q}_{0}\right)$ is chosen as the desired state $\left(q_{d}, \dot{q}_{d}\right)$, the integral curve of the single-dimensional dynamic system is the desired trajectory.

In differential equation (15), different initial states are corresponding to different integration curves. To ensure the uniqueness of the trajectory, parameters describing the curve are needed. Let $Y\left(q_{2}\right)=\dot{q}_{2}^{2} ;(15)$ can be converted to

$$
\frac{d Y}{d q_{2}}+\sigma_{0}\left(q_{2}\right) Y-\omega_{0}\left(q_{2}\right)=0
$$

where

$$
\begin{aligned}
& \sigma_{0}=2 \frac{\sigma\left(q_{2}\right)}{\eta\left(q_{2}\right)}, \\
& \omega_{0}=-2 \frac{\omega\left(q_{2}\right)}{\eta\left(q_{2}\right)} .
\end{aligned}
$$


By introducing the integration factor

$$
I=e^{\int_{q_{0}}^{q_{2}} \sigma_{0}(x) d x}
$$

the solution of the $Y\left(q_{2}\right)$ is derived as

$$
\begin{aligned}
& Y\left(q_{2}\right)=I^{-1} \int_{q_{0}}^{q_{2}} I \omega_{0}(x) d x+Y_{0} I^{-1} \\
& =-\frac{a(M+m)}{\eta^{2}\left(q_{2}\right)}\left(q_{2}-q_{0}\right)\left[\omega\left(q_{2}\right)+\omega\left(q_{0}\right)\right] \\
& +\frac{\eta^{2}\left(q_{0}\right)}{\eta^{2}\left(q_{2}\right)} \dot{q}_{0}^{2} \\
& +\frac{2}{\eta^{2}\left(q_{2}\right)}[(b k+(M+m) g \cos \gamma \sin \beta) \\
& \left.+\left(\sigma\left(q_{2}\right)-\sigma\left(q_{0}\right)\right)\right]+\frac{2 a k}{\eta^{2}\left(q_{2}\right)}\left[\dot{\sigma}\left(q_{2}\right)-\dot{\sigma}\left(q_{0}\right)\right] \\
& +\frac{2}{\eta^{2}\left(q_{2}\right)}\left[\omega\left(q_{2}\right) \sigma\left(q_{2}\right)-\omega\left(q_{0}\right) \sigma\left(q_{0}\right)\right] .
\end{aligned}
$$
as

Consequently, the trajectory function $\psi$ can be denoted

$$
\psi\left(q_{2}, \dot{q}_{2}, q_{0}, \dot{q}_{0}\right)=\dot{q}_{2}^{2}-Y\left(q_{2}\right)
$$

\section{Trajectory Tracking Control}

The generated trajectory is determined by both virtual constraint (13) and orbit function (21). To ensure that the inclined TORA system converges to the target trajectory, the trajectory tracking control can be converted to an output stabilization issue. And virtual constraint equation (13) can be denoted as

$$
\rho=\varphi\left(q_{2}\right)-q_{1} \text {. }
$$

In this section, cascade control method will be employed to realize stabilization of system (21) and (22). The virtual constraint is regarded as the inner loop of the closed-loop system, while the orbit trajectory is taken as the outer loop.

4.1. The Stabilization Controller Design of Virtual Constraint. For the inclined TORA system, by neglecting the disturbance force Matrix N, compact matrix form (4) can be rewritten as

$$
\begin{aligned}
& m_{11} \ddot{q}_{1}+m_{12} \ddot{q}_{2}+h_{1}=0, \\
& m_{21} \ddot{q}_{1}+m_{22} \ddot{q}_{2}+h_{2}=\tau,
\end{aligned}
$$

where

$$
\begin{aligned}
& h_{1}=-m r \sin q_{2} \dot{q}_{2}^{2}+k q_{1}+(M+m) g \cos \gamma \sin \beta, \\
& h_{2}=m g r \cos \left(\gamma+q_{2}\right) \sin \beta .
\end{aligned}
$$

Consequently, $\ddot{q}_{1}$ and $\ddot{q}_{2}$ can be calculated as

$$
\begin{aligned}
& \ddot{q}_{1}=\frac{-m_{12} \tau-m_{22} h_{1}+m_{12} h_{2}}{m_{11} m_{22}-m_{12} m_{21}} \triangleq g_{1} \tau+f_{1}, \\
& \ddot{q}_{2}=\frac{m_{11} \tau+m_{21} h_{1}-m_{11} h_{2}}{m_{11} m_{22}-m_{12} m_{21}} \triangleq g_{2} \tau+f_{2} .
\end{aligned}
$$

Combining selected virtual constraint (13) and subsystem (22) and by differentiating both sides of the equation, the output function of the virtual constraint can be expressed as

$$
\begin{aligned}
& \rho=a q_{2}+b-q_{1}, \\
& \dot{\rho}=a \dot{q}_{2}-\dot{q}_{1}, \\
& \ddot{\rho}=a \ddot{q}_{2}-\ddot{q}_{1} .
\end{aligned}
$$

To construct the relationship among each equation in (28), a virtual control input $v$ is designed and introduced as

$$
\nu=a f_{2}+a g_{2} \tau-f_{1}-g_{1} \tau+k_{1} \dot{\rho}+k_{2} \rho,
$$

where $k_{1}$ and $k_{2}$ are positive constants needed to be decided. Combining inclined TORA system (26) and (29), the innerloop subsystem can be written as

$$
\ddot{\rho}+k_{1} \dot{\rho}+k_{2} \rho=\nu .
$$

According to Laplace transformation, the transfer function of the subsystem can be rewritten as

$$
G(s)=\frac{\rho(s)}{\nu(s)}=\frac{1}{s^{2}+k_{1} s+k_{2}} .
$$

Therefore, by appropriate selecting of parameters $k_{1}$ and $k_{2}$, the input and output stabilization of the subsystem can be achieved.

4.2. The Stabilization Controller Design of Periodic Trajectory. For virtual constraint (13), assuming that the target rate for the state is 0 , that is, $Y_{0}=0$, the orbit equation can be written as

$$
\psi\left(q_{2}, \dot{q}_{2}, q_{0}, \dot{q}_{0}\right)=\dot{q}_{2}^{2}-I^{-1} \int_{q_{0}}^{q_{2}} I \omega_{0} d x
$$

A simple control Lyapunov function (CLF) can be selected as

$$
V=\frac{1}{2} \psi^{2}
$$

By differentiating the CLF, one can obtain

$$
\dot{V}=\psi \dot{q}_{2}\left(2 \ddot{q}_{2}+I^{-1} \sigma_{0} \int_{q_{0}}^{q_{2}} I \omega_{0} d x-\omega_{0}\right) \text {. }
$$

Combining (23), (28), and (30), $\ddot{q}_{2}$ can be expressed as

$$
\ddot{q}_{2}=\frac{m_{11} v-m_{11}\left(k_{1} \dot{\rho}+k_{2} \rho\right)-h_{1}}{a m_{11}+m_{12}} .
$$

Substituting (35) into (34), we have

$$
\dot{V}=\psi \dot{q}_{2}[g(\cdot) \nu+f(\cdot)]
$$

where

$$
\begin{aligned}
f(\cdot)= & -2 \frac{m_{11}\left(k_{1} \dot{\rho}+k_{2} \rho\right)+h_{1}}{a m_{11}+m_{12}}+I^{-1} \sigma_{0} \int_{q_{0}}^{q_{2}} I \omega_{0} d x \\
& -\omega_{0}, \\
g(\cdot)= & 2 \frac{m_{11}}{a m_{11}+m_{12}} .
\end{aligned}
$$


By selecting the following control input torque $\tau$

$$
\nu=-\frac{k_{3}|\psi| \operatorname{sgn}\left(\psi \dot{q}_{2}\right)+f(\cdot)}{g(\cdot)},
$$

where $k_{3}$ is a positive constant and $\operatorname{sgn}(\cdot)$ is a signal function, we have

$$
\dot{V}=-k_{3}|\psi| \psi \dot{q}_{2} \operatorname{sgn}\left(\psi \dot{q}_{2}\right) \leq 0 .
$$

Therefore, according to second stability theorem of Lyapunov [4], the trajectory tracking control of the system can be realized asymptotically.

Combing (29) and (38), the physical control input $\tau$ can be calculated as

$$
\begin{aligned}
\tau= & \frac{-\left(k_{3}|\psi| \operatorname{sgn}\left(\psi \dot{q}_{2}\right)+f(\cdot)\right) / g(\cdot)}{a g_{2}-g_{1}} \\
& -\frac{k_{1} \dot{\rho}+k_{2} \rho+a f_{2}-f_{1}}{a g_{2}-g_{1}} .
\end{aligned}
$$

\section{Simulations}

To verify the dynamical analysis and control design for the inclined TORA system, simulations were programmed and performed with MATLAB/Simulink. Following [1], the physical parameters of the inclined TORA system are chosen as shown in Table 1 . The controller parameters are selected as follows: $k_{1}=20, k_{2}=400$, and $k_{3}=10$. For the inclined TORA system, we choose a typical case to study, which is $\beta=45^{\circ}$ and $\gamma=45^{\circ}$; namely, the TORA system is on representational inclined plane. The desired point is $(x, \theta, \dot{x}, \dot{\theta})=(0.1, \pi / 2,0,0)$. According to (7) and (8), one can get the equilibriums of the system as $(0.0383554, \pi / 4,0,0)$. For the given starting and desired points, virtual constraint (13) can be calculated as

$$
x=0.07848834244 \theta-0.0232892 .
$$

To ensure that the rotor can start up easily, we choose the initial point of the system as $(0,0.1,0,0)$. Based on the above conditions, the simulation results are shown in Figure 2. From the simulation results, one can see that the inclined TORA system oscillates to the desired state after about 10 seconds, periodically. At the beginning of the oscillation from the initial point, the control input torque $\tau$ drives both the cart position $x$ and rotor angle $\theta$ oscillating with increasing amplitudes. During the translational position of the cart $x$ and the angular position of the rotor $\theta$ approaching the desired trajectory from 4 seconds to 8 seconds, the amplitude of control input torque $\tau$ remains smooth. And the control input torque $\tau$ has a small mutation in the oscillation at about 8 seconds as shown in the right top subfigure because the cart position $x$ has reached the desired point while the rotor angle has not until about 2 seconds later. And the maximum control torque is around $0.2 \mathrm{~N} \cdot \mathrm{m}$. Once the translational position of the cart $x$ and the angular position of the rotor $\theta$ converge to the target trajectory, gradually, the control input torque $\tau$ runs periodically to guarantee the trajectory tacking control.
TABLE 1: Physical parameters of the TORA system.

\begin{tabular}{lcc}
\hline $\begin{array}{l}\text { Parameter } \\
\text { (units) }\end{array}$ & Value & Description \\
\hline$M(\mathrm{~kg})$ & 1.3608 & Cart mass of axis $x$ \\
$m(\mathrm{~kg})$ & 0.096 & Rotor mass \\
$k(\mathrm{~N} / \mathrm{m})$ & 186.3 & Spring stiffness of axis $x$ \\
$r(\mathrm{~m})$ & 0.0592 & Eccentric distance of rotor \\
$I\left(\mathrm{~kg} \cdot \mathrm{m}^{2}\right)$ & 0.0002175 & Rotor inertia \\
\hline
\end{tabular}

Due to the inclined plane, the trajectory of the ball in plane oxyz is with asymmetric periodic motion as shown in the right bottom subfigure.

Another simulation virtual constraint based control design for the inclined TORA system was done with the same physical parameters and desired trajectory point $(x, \theta, \dot{x}, \dot{\theta})=$ $(0.1, \pi / 2,0,0)$, while the parameters of the inclined amplitude and direction were selected as $\beta=30^{\circ}$ and $\gamma=30^{\circ}$, respectively. The equilibriums of the system are calculated as $(0.0332167, \pi / 3,0,0)$, and new virtual constraint (13) can be calculated as

$$
x=0.1275466731 \theta-0.10034985 .
$$

The simulation results are shown in Figure 3. According to Figure 3, one can see that about 20 seconds is needed for the inclined TORA system to oscillate to the desired state, which is much slower than the case with $\beta=45^{\circ}$ and $\gamma=45^{\circ}$ as shown in Figure 2. In other words, the convergence speed of the oscillation is related to the inclined angles of the slope. At the beginning of the oscillation from the initial point, the distortion of the phase trajectory of the rotor is more serious as shown in the left bottom subfigure. The control input torque $\tau$ increases over time smoothly at the beginning and achieves periodical stability finally. Although the angles of the inclined plane are changed, since the TORA system is still on an inclined plane, the trajectory of the rotor in plane oxyz keeps asymmetry as shown in the right bottom subfigure.

For the special case of the benchmark TORA, which is with $\beta=0^{\circ}$ of the inclined plane, the TORA system is on a horizontal plane. The desired point is also selected as $(x, \theta, \dot{x}, \dot{\theta})=(0.1, \pi / 2,0,0)$. However, in this case, angle equilibrium (8) of the TORA system can be arbitrary. Typically, we can choose the equilibriums as $(0,0,0,0)$. For given starting and desired points above, virtual constraint (13) can be calculated as

$$
x=\frac{0.2}{\pi} \theta .
$$

The simulation results are shown in Figure 4. From the simulation results, the system oscillates to the desired state after about 28 seconds, which is slower than those on inclined planes. In this special case, the oscillation of the system will not be influenced by the gravity $g$, and the symmetry trajectories of the rotor are achieved. The reason for the longer stability time is because the initial point of the system $(0,0.1,0,0)$ is closer to the equilibriums of the TORA system, 

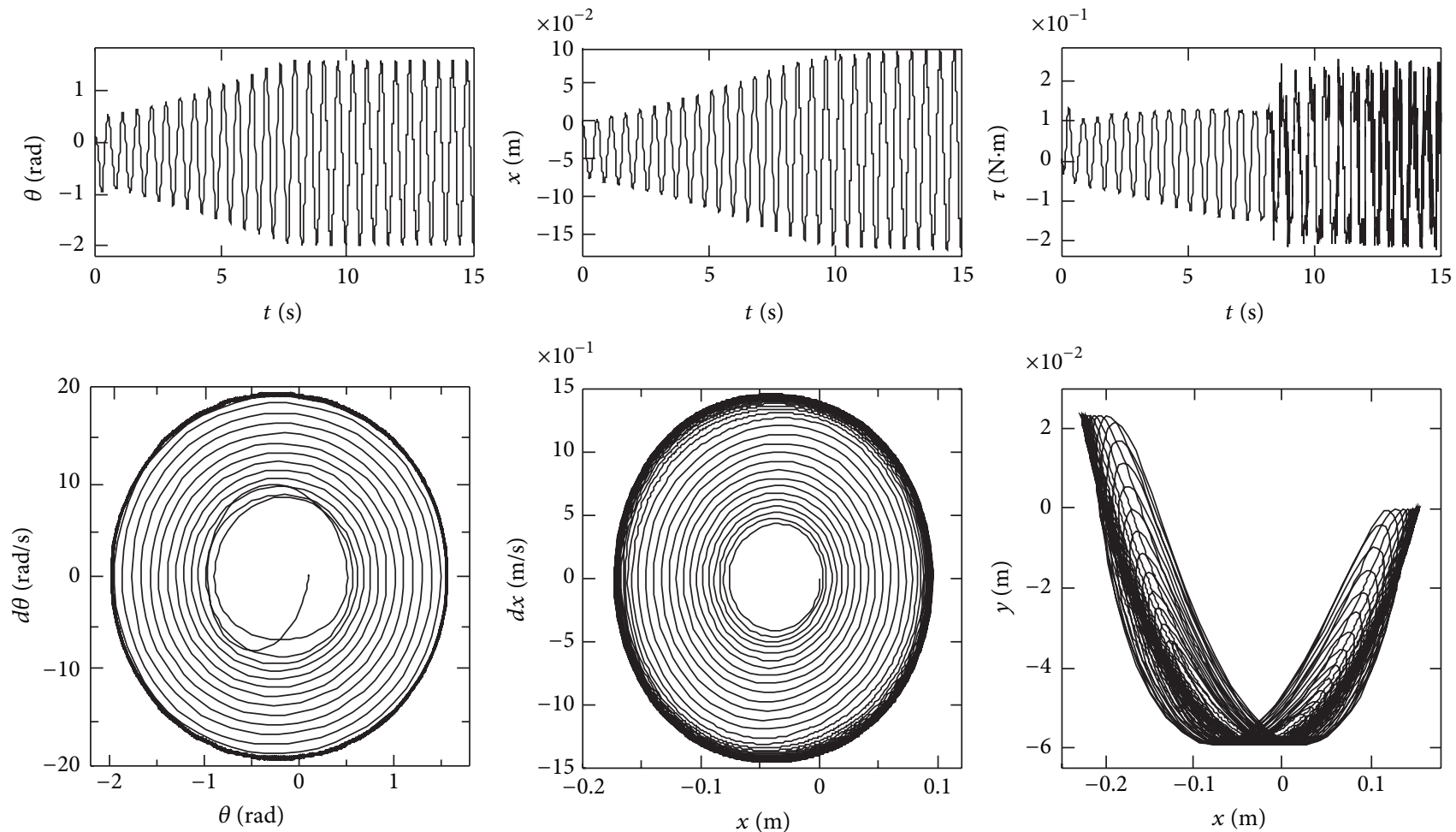

Figure 2: Simulation results of TORA system on the inclined plane, $\beta=45^{\circ}, \gamma=45^{\circ}$.
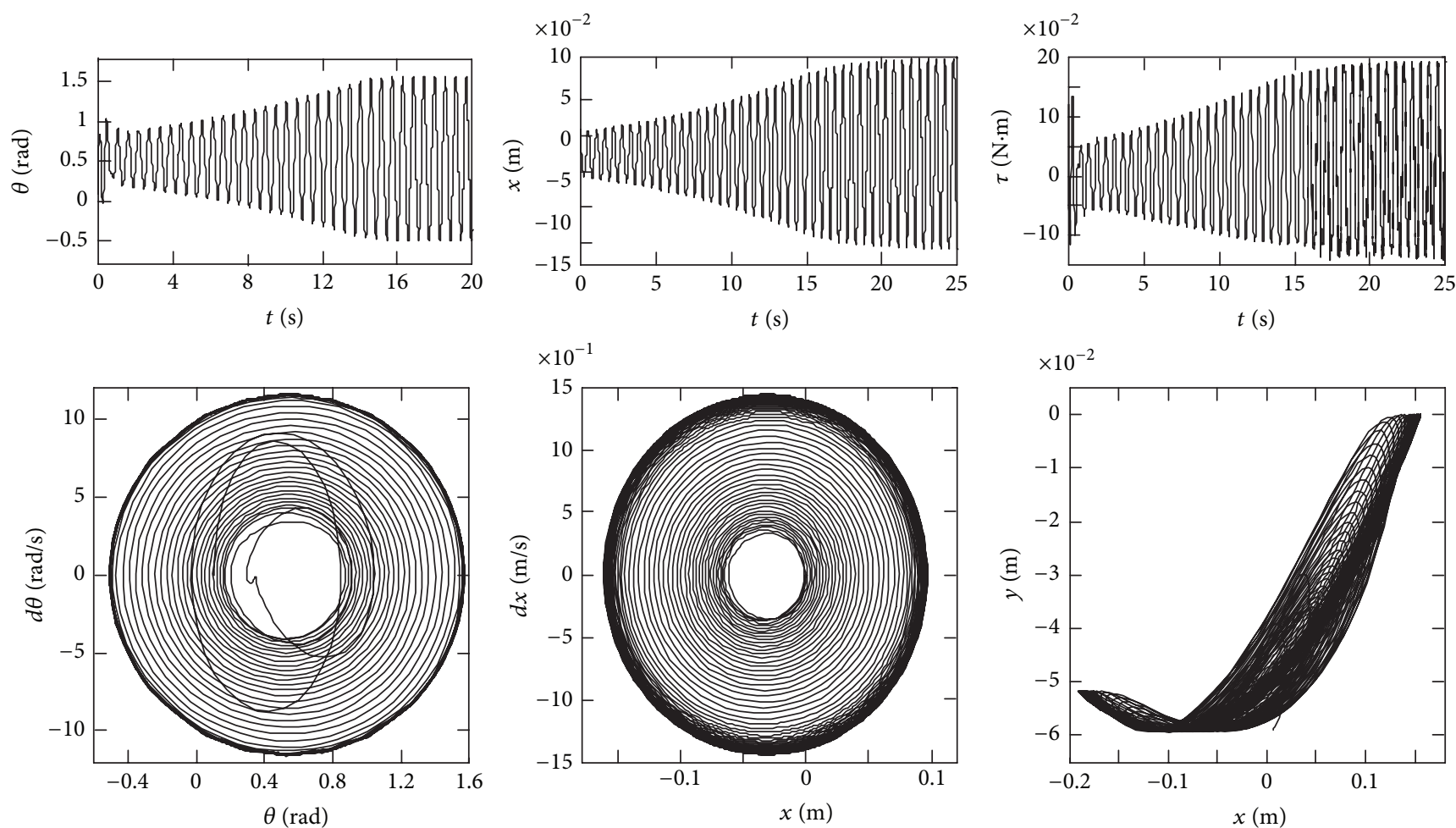

FIgURE 3: Simulation results of TORA system on the inclined plane, $\beta=30^{\circ}, \gamma=30^{\circ}$. 

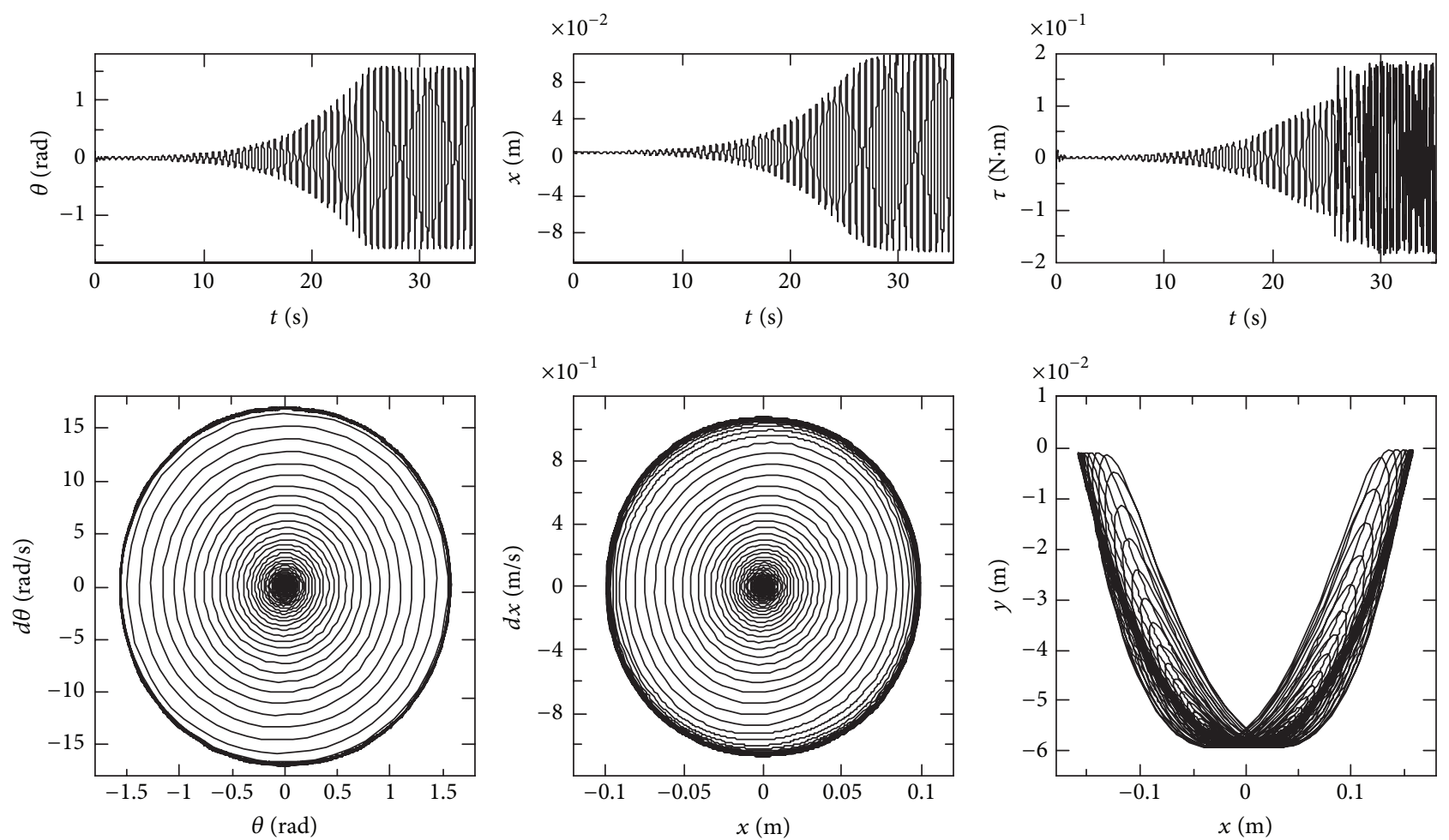

FiguRE 4: Simulation results of TORA system on the horizontal plane, $\beta=0^{\circ}, \gamma=0^{\circ}$.

which leads to smaller control input torque $\tau$ at the beginning. It can been seen from Figure 4 that the control input increases very slowly at the beginning and the trajectories of the rotor and the cart stay at around the centers of the phase planes at the beginning of their oscillating motions.

As a result, the simulation results verified that the proposed virtual constraints based control design can track the trajectories passing through desired points in the state space of the inclined TORA system successfully.

\section{Conclusions}

In this paper, steering an underactuated TORA system on a slop to its arbitrary configuration point is studied. A virtual constraint based trajectory generation scheme is designed to generate periodic trajectories that pass through arbitrary points in the state space. A cascade control method is presented to stabilize trajectory tracking control of the converted dynamic system combing the virtual constraint and periodic trajectory, where the virtual constraint and periodic trajectory are taken as the inner loop and outer loop of the close-loop system, respectively. Simulation results show that the proposed control design can steer the TORA system to the generated trajectories passing through desired points successfully. And for the inclined TORA system, the period trajectories of the rotor in its motion plane will be asymmetric while those are symmetric for horizontal TORA system, which arises from the gravity effect or not. The methods proposed in this paper can also be applied to other underactuated mechanical systems to realize complex tasks and extend their applications.

\section{Conflict of Interests}

The authors declare that there is no conflict of interests regarding the publication of this paper.

\section{Acknowledgments}

The work is financially supported by National Science Foundation of China (11102039), the Excellent Young Teachers Program of Southeast University (2242015R30024), and Six Talent Peaks Project of Jiangsu Province (2014-ZBZZ-001).

\section{References}

[1] R. T. Bupp, D. S. Bernstein, and V. T. Coppola, "A benchmark problem for nonlinear control design," International Journal of Robust and Nonlinear Control, vol. 8, no. 4-5, pp. 307-310, 1998.

[2] M. W. Spong, "Underactuated mechanical systems," in Control Problems in Robotics and Automation, B. Siciliano and K. P. Valavanis, Eds., vol. 230 of Lecture Notes in Control and Information Sciences, pp. 135-150, Springer, Berlin, Germany, 1998.

[3] M. Reyhanoglu, A. van der Schaft, N. H. McClamroch, and I. Kolmanovsky, "Dynamics and control of a class of underactuated mechanical systems," IEEE Transactions on Automatic Control, vol. 44, no. 9, pp. 1663-1671, 1999.

[4] I. Fantoni and R. Lozano, Nonlinear Control for Underactuated Mechanical Systems, Springer, London, UK, 2002.

[5] R. T. Bupp, D. S. Bernstein, and V. T. Coppola, "Experimental implementation of integrator backstepping and passive nonlinear controllers on the RTAC testbed," International Journal of Robust and Nonlinear Control, vol. 8, no. 4-5, pp. 435-457, 1998. 
[6] P. Tsiotras, M. Corless, and M. A. Rotea, "An L2 disturbance attenuation solution to the nonlinear benchmark problem," International Journal of Robust and Nonlinear Control, vol. 8, no. 4-5, pp. 311-330, 1998.

[7] M. Jankovic, D. Fontaine, and P. V. Kokotović, “TORA example: cascade- and passivity-based control designs," IEEE Transactions on Control Systems Technology, vol. 4, no. 3, pp. 292-297, 1996.

[8] C.-H. Lee and S.-K. Chang, "Experimental implementation of nonlinear TORA system and adaptive backstepping controller design," Neural Computing and Applications, vol. 21, no. 4, pp. 785-800, 2012.

[9] Z. Petres, P. Baranyi, and H. Hashimoto, "Approximation and complexity trade-off by TP model transformation in controller design: a case study of the TORA system," Asian Journal of Control, vol. 12, no. 5, pp. 575-585, 2010.

[10] J. She, A. Zhang, X. Lai, and M. Wu, "Global stabilization of 2DOF underactuated mechanical systems - an equiavlent-inputdisturbance approach," Nonlinear Dynamics, vol. 69, no. 1-2, pp. 495-509, 2012.

[11] T. Burg and D. Dawson, "Additional notes on the TORA example: a filtering approach to eliminate velocity measurements," IEEE Transactions on Control Systems Technology, vol. 5, no. 5, pp. 520-523, 1997.

[12] G. Escobar, R. Ortega, and H. Sira-Ramírez, "Output-feedback global stabilization of a nonlinear benchmark system using a saturated passivity-based controller," IEEE Transactions on Control Systems Technology, vol. 7, no. 2, pp. 289-293, 1999.

[13] F. Celani, "Output regulation for the TORA benchmark via rotational position feedback," Automatica, vol. 47, no. 3, pp. 584-590, 2011.

[14] B. T. Gao, "Dynamical modeling and energy-based control design for TORA," Acta Automatica Sinica, vol. 34, no. 9, pp. 1221-1224, 2008

[15] J. M. Avis, S. G. Nersesov, R. Nathan, H. Ashrafiuon, and K. R. Muske, "A comparison study of nonlinear control techniques for the RTAC system," Nonlinear Analysis: Real World Applications., vol. 11, no. 4, pp. 2647-2658, 2010.

[16] B. Gao and F. Ye, "Dynamical analysis and stabilizing control of inclined rotational translational actuator systems," Journal of Applied Mathematics, vol. 2014, Article ID 598384, 9 pages, 2014.

[17] B. Gao, J. Xu, J. Zhao, and X. Huang, "Stabilizing control of an underactuated 2-dimensional tora with only rotor angle measurement," Asian Journal of Control, vol. 15, no. 5, pp. 14771488, 2013.

[18] A. Shiriaev, J. W. Perram, and C. Canudas-de-Wit, "Constructive tool for orbital stabilization of underactuated nonlinear systems: virtual constraints approach," IEEE Transactions on Automatic Control, vol. 50, no. 8, pp. 1164-1176, 2005.

[19] C. C. Chung and J. Hauser, "Nonlinear control of a swinging pendulum," Automatica, vol. 31, no. 6, pp. 851-862, 1995.

[20] A. Shiriaev, A. Robertsson, J. Perram, and A. Sandberg, "Periodic motion planning for virtually constrained Euler-Lagrange systems," Systems \& Control Letters, vol. 55, no. 11, pp. 900-907, 2006.

[21] A. S. Shiriaev, L. B. Freidovich, A. Robertsson, R. Johansson, and A. Sandberg, "Virtual-holonomic-constraints-based design of stable oscillations of Furuta pendulum: theory and experiments," IEEE Transactions on Robotics, vol. 23, no. 4, pp. 827$832,2007$.
[22] P. X. La Hera, L. B. Freidovich, A. S. Shiriaev, and U. Mettin, "New approach for swinging up the furuta pendulum: theory and experiments," Mechatronics, vol. 19, no. 8, pp. 1240-1250, 2009.

[23] H. Cheng, H. Chen, X. Zhang, B. Gao, and H. Chen, "Periodic trajectory generation and tracking control for a class of underactuated mechanical systems," in Proceedings of the IEEE International Conference on Robotics and Biomimetics, pp. 18171822, Guangzhou, China, December 2012. 

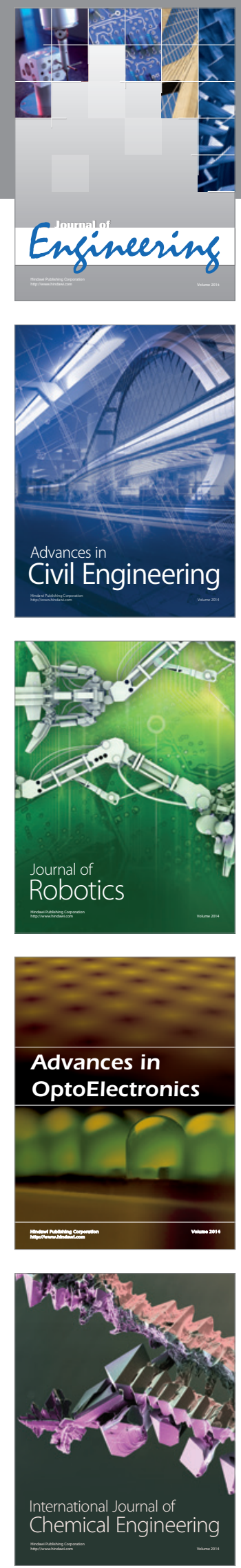

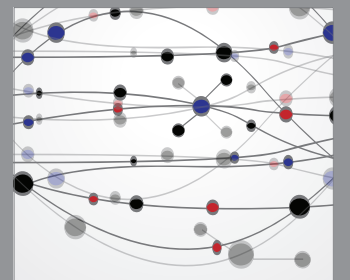

The Scientific World Journal
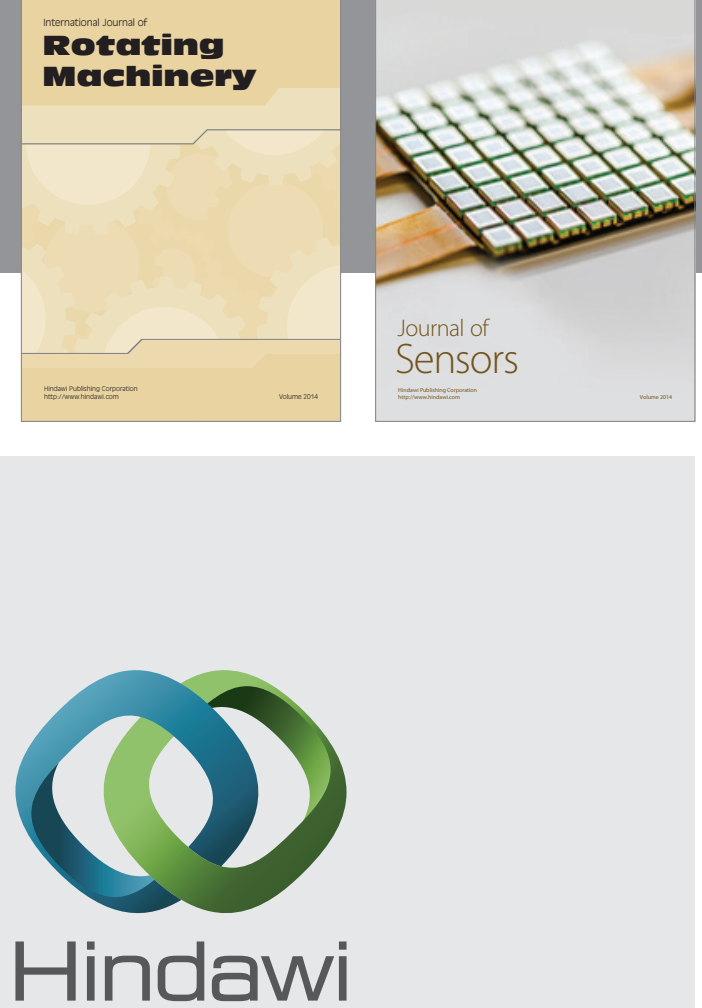

Submit your manuscripts at http://www.hindawi.com
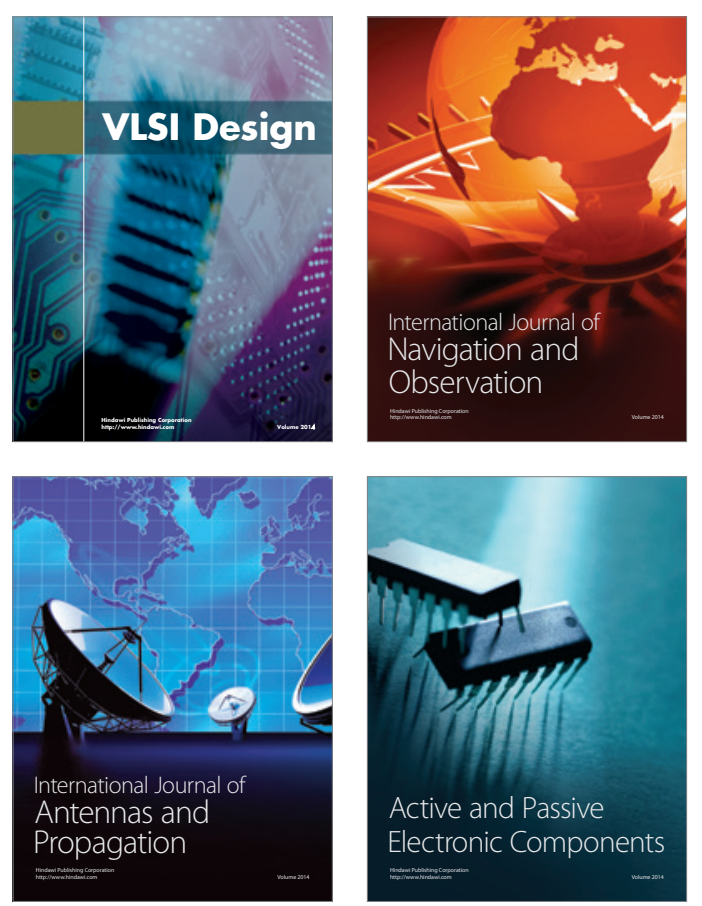
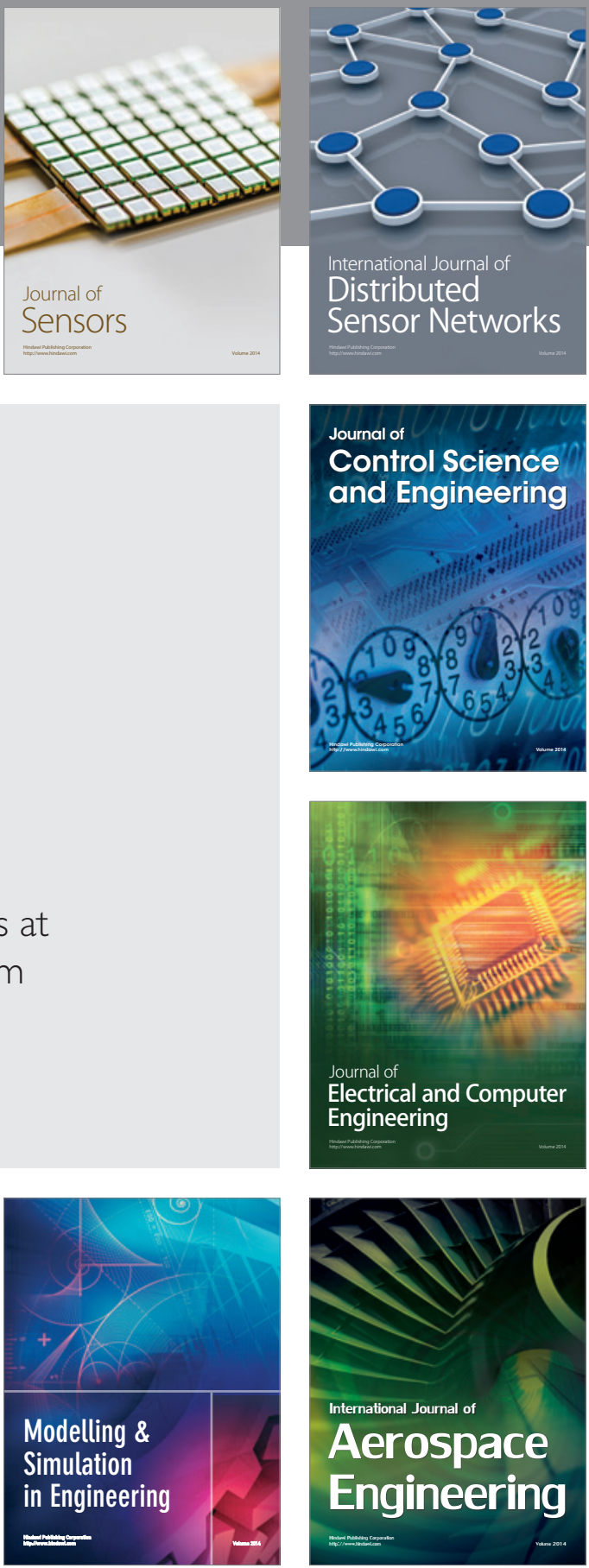

Journal of

Control Science

and Engineering
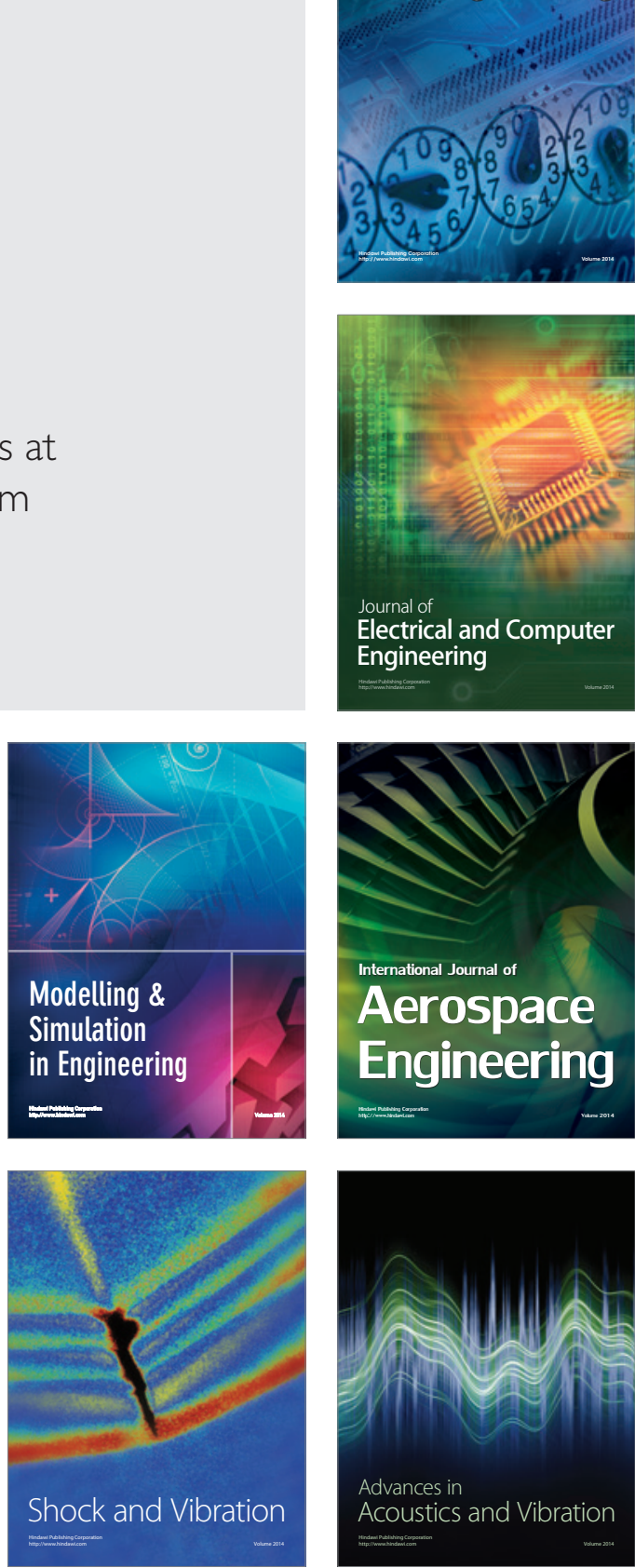